\title{
On Motion Mechanism Calibration Technology of a Linear Structured Light 3D Measurement System
}

\author{
Kui Wang \\ Automation Research Center \\ Dalian Maritime University \\ Dalian, China \\ roy_wangkui@sina.com \\ Ying $\mathrm{Hu}$ \\ Automation Research Center \\ Dalian Maritime University \\ Dalian, China \\ hawk_huy@sina.com
}

\author{
Fenglong Bi \\ Automation Research Center \\ Dalian Maritime University \\ Dalian, China \\ bifenglong08@163.com \\ Yue Ma \\ Automation Research Center \\ Dalian Maritime University \\ Dalian, China \\ mayue.1881@163.com
}

\begin{abstract}
Aiming at the motion mechanism calibration of a 2DOF linear structured light three-dimensional (3D) measurement system, a significant method is proposed in this article. Firstly, the physical model of the measurement system is given. Secondly, the calibration model is deduced and a calibration method based on high-low ball is presented. In order to compensate mechanical errors existing in the system, an iterative data correction algorithm is put forward. The experimental results shown that the accuracy of the measurement system can be greatly improved by this algorithm.
\end{abstract}

Keywords- linear structured light measurement; motion mechanism calibration; iterative data correction; $3 D$ point cloud

\section{INTRODUCTION}

Linear structured light measurement technology has been widely used in many areas of industrial and civil, greatly promote the further development of related fields. Because it characteristics that simple structure, good antijamming, high precision and wide range of applications [1], it has occupied a major position in the field of structured light measurement. For the $2 \mathrm{DOF}$ linear structured light 3D measurement system introduced in this paper, a special method of calibrating the motion mechanism is proposed. A further study on how to improve the measurement precision is conducted as some errors do exist in the measurement mechanism.

\section{RELATED RESEARCH}

The principle of linear structured light measurement is as following: a linear structured light source emits a ray to the surface of measured object, due to limited by the shape of the object, the camera record is a curved stripe, through the curved stripe, 3D contour data of a crosssection of the object can be obtained[2]. As a result of only one line measurement data can be obtained one time, it has to rely on motion mechanism to complete the whole 3D measurement[3].
Many works have been done on the measurement system calibration, the most typical methods are: tooth target method [4], constant cross-ratio method [5] and onedimensional coordinate method [6], etc. However, the calibration of the linear structured light 3D measurement system proposed in this paper is on the basis of adopting high-low ball method, meanwhile, for the purpose of measuring more accurate, an iterative data correction algorithm is presented. From the experimental results, this algorithm can effectively improve the measurement system precision.

\section{PRINCIPLE INTRODUCTION}

The 3D measurement system consists of a rotating turntable and an elevating axis which contains a linear structured light probe, in addition, the linear structure light probe is composed by a camera and a light source (Figure.1). Put a measured object on the rotating turntable, the camera records the irradiated light on measured object. After calibration, the 3D coordinates of the current light can be obtained, then utilize the probe to move up and down on elevating axis and rotate the turntable to acquire the whole 3D information of the object's surface.

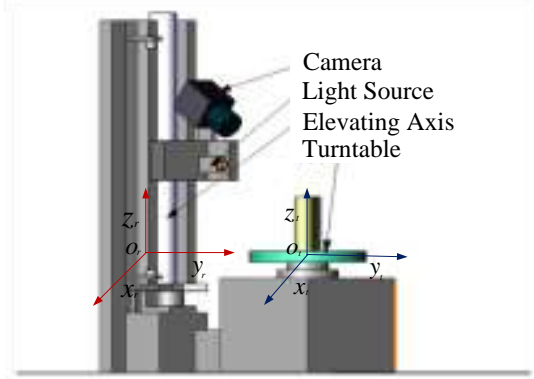

Figure 1. Schematic plan of the 2DOF linear structured light 3D measurement system.

Adjust the angle of the linear structured light source to make light plane perpendicular to the elevating axis. Establish a probe coordinate syste $O_{r}-X_{r} Y_{r} Z_{r}$ as Figure. 1 
shows by light plane and elevating axis, then establish a turntable coordinate system $O_{t}-X_{t} Y_{t} Z_{t}$ by the turntable plane and an axis perpendicular to the turntable plane. After the probe scans measured object's one side from bottom to top, the turntable will rotate a certain angle and the probe scan the object's another side from bottom to top until the turntable rotates one circle for the probe scanning the entire surface.

\section{A. Linear structured light probe calibration}

There are many methods of calibrating linear structured light probe, among them the typical representative methods are: two-step [7], dual-plane method [8], Zhang Zhengyou calibration method [9], DLT (Direct Linear Transformation) [10], natural calibration method and so on. This paper adopted the previous laboratory research result: an improved two-step calibration method. The result of the calibration is establishing a probe coordinate system $O_{r}-X_{r} Y_{r} Z_{r}$ using light plane as $Z=0$ plane. In this case, the current stripe coordinates $x_{r}$ and $y_{r}$ at probe coordinate system can be directly obtained, thus the coordinate $z_{r}$ can be obtained through the probe motion on elevating axis. After the linear structured light probe calibration, all date acquired are the object's 3D coordinates at probe coordinate system. For more details of the calibration method, please refer to literature [11].

\section{B. Motion mechanism calibration}

The main purpose of motion mechanism calibration is to search the transformation from probe coordinate system to turntable coordinate system, namely, to compute rotation matrix $R$ and translation matrix $T$. The following process is using high-low ball method to compute $R$ and $T$.

1) Fix a ball on the turntable, the probe scans the ball to obtain the position of a spherical center, then the turntable rotates a certain angle, the probe scans the ball again to obtain the second spherical center position. Multiple spherical center positions can be obtained after the turntable rotates one circle, calculate the circle center coordinate $C_{m}\left(x_{m}, y_{m}, z_{m}\right)$ which is fitted by all spherical centers.

2) Rise the ball to a certain height and repeat the first step to acquire the second fitting circle center $C_{n}\left(x_{n}, y_{n}, z_{n}\right)$. Obviously, a line through the two fitting circle centers is the $O_{t} Z_{t}$-axis of turntable coordinate system. (Figure.2)

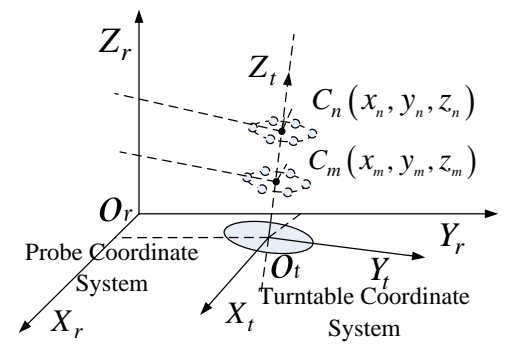

Figure 2. Schematic diagram of fitting circle centers using high-low ball method.
3) After many times measurement, compute the average value, according to straight line-space equation:

$$
\frac{x-x_{m}}{x_{n}-x_{m}}=\frac{y-y_{m}}{y_{n}-y_{m}}=\frac{z-z_{m}}{z_{n}-z_{m}}
$$

Calculate the intersection $O_{t}\left(x_{t}, y_{t}, z_{t}\right)$ of $O_{t} Z_{t}$-axis with $X_{r} O_{r} Y_{r}$-plane, in that way, the position of turntable coordinate system relative to probe coordinate system is determined. Hence the translation matrix is

$$
T=\left[\begin{array}{c}
x_{t} \\
y_{t} \\
0
\end{array}\right]
$$

Assume the unit vector of $O_{t} Z_{t}$-axis is $\vec{m}(a, b, c)$ at probe coordinate system, if probe coordinate system $O_{r}-X_{r} Y_{r} Z_{r}$ can be converted to turntable coordinate system $O_{t}-X_{t} Y_{t} Z_{t}$ through $\alpha$ angle of rotation around $O_{r} X_{r}$-axis, $\beta$ angle of rotation around $O_{r} Y_{r}$-axis and $\gamma$ angle of rotation around $O_{r} Z_{r}$-axis, then

$$
\left\{\begin{array}{cc}
\sin \alpha=\frac{-b}{\sqrt{b^{2}+c^{2}}} & \cos \alpha=\frac{c}{\sqrt{b^{2}+c^{2}}} \\
\sin \beta=\frac{a}{\sqrt{a^{2}+c^{2}}} & \cos \beta=\frac{c}{\sqrt{a^{2}+c^{2}}} \\
\sin \gamma=0 & \cos \gamma=1
\end{array}\right.
$$

According to the rotation matrix formula

$R=\left[\begin{array}{ccc}\cos \beta \cos \gamma & \sin \alpha \sin \beta \cos \gamma-\cos \alpha \sin \gamma & \cos \alpha \sin \beta \cos \gamma+\sin \alpha \sin \gamma \\ \cos \beta \sin \gamma & \sin \alpha \sin \beta \sin \gamma+\cos \alpha \cos \gamma & \cos \alpha \sin \beta \sin \gamma-\sin \alpha \cos \gamma \\ -\sin \beta & \sin \alpha \cos \beta & \cos \alpha \cos \beta\end{array}\right]$

Consequently, the rotation matrix

$$
R=\left[\begin{array}{ccc}
\frac{c}{\sqrt{a^{2}+c^{2}}} & \frac{-a b}{\sqrt{\left(a^{2}+c^{2}\right)\left(b^{2}+c^{2}\right)}} & \frac{a c}{\sqrt{\left(a^{2}+c^{2}\right)\left(b^{2}+c^{2}\right)}} \\
0 & \frac{c}{\sqrt{b^{2}+c^{2}}} & \frac{b}{\sqrt{b^{2}+c^{2}}} \\
\frac{-a}{\sqrt{a^{2}+c^{2}}} & \frac{-b c}{\sqrt{\left(a^{2}+c^{2}\right)\left(b^{2}+c^{2}\right)}} & \frac{c^{2}}{\sqrt{\left(a^{2}+c^{2}\right)\left(b^{2}+c^{2}\right)}}
\end{array}\right]
$$

4) For one point $P_{t 0}\left(x_{t 0}, y_{t 0}, z_{t 0}\right)$ on measured object, the coordinate of it at probe coordinate system is $P_{r}\left(x_{r}, y_{r}, z_{r}\right)$.After $\theta$ angle of counterclockwise rotation for the turntable, the real coordinate of the point is $P_{t 1}\left(x_{t 1}, y_{t 1}, z_{t 1}\right)$, utilize rotation matrix $R$ and translation matrix $T$ to obtain

$$
\left[\begin{array}{l}
x_{t 1} \\
y_{t 1} \\
z_{t 1}
\end{array}\right]=\left[\begin{array}{ccc}
\cos \theta & -\sin \theta & 0 \\
\sin \theta & \cos \theta & 0 \\
0 & 0 & 1
\end{array}\right]\left(R\left[\begin{array}{l}
x_{r} \\
y_{r} \\
z_{r}
\end{array}\right]+T\right)
$$

\section{Errors compensation for the measuring mechanism}

In front, we assume probe coordinate system of the 3D measurement system as world coordinate system, convert all measurement data at world coordinate system to turntable coordinate system to achieve $3 \mathrm{D}$ reconstruction of the object through rotation matrix $R$ and translation 
matrix $T$. However, as a result of mechanical craft deviations, the elevating axis is not strictly perpendicular to light plane, if the light plane at the bottom of elevating axis is selected to establish world coordinate system $O_{w}-X_{w} Y_{w} Z_{w}$ as shown in Figure.3, there will be some offsets between elevating axis and $O_{w} Z_{w}$-axis.

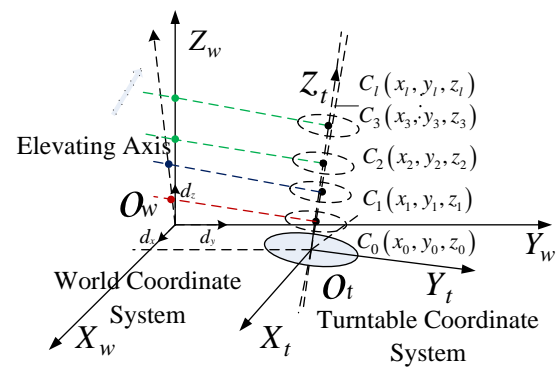

Figure 3. Schematic plan of the iterative data correction

Assume the unit vector of elevating axis is $\vec{n}\left(d_{x}, d_{y}, d_{z}\right)$ at world coordinate system, for point $P$ on measured object, the obtained coordinate $P_{w}\left(x_{w}, y_{w}, z_{w}\right)$ at world coordinate system is not the actual coordinate. The real actual coordinate of point $P$ is $P_{w}^{\prime}\left(x_{w}^{\prime}, y_{w}^{\prime}, z_{w}^{\prime}\right)$, moreover

$$
\left\{\begin{array}{l}
x_{w}^{\prime}=x_{w}+z_{w} d_{x} \\
y_{w}^{\prime}=y_{w}+z_{w} d_{y} \\
z_{w}^{\prime}=z_{w} d_{z}
\end{array}\right.
$$

In order to calculate $\vec{n}\left(d_{x}, d_{y}, d_{z}\right)$, high-low ball calibration method of the motion mechanism is still chosen.

Step 1: Put the ball on the turntable two different heights about $10 \mathrm{~mm}$ and $20 \mathrm{~mm}$ respectively for scanning, two circle centers $C_{0}\left(x_{0}, y_{0}, z_{0}\right)$ and $C_{1}\left(x_{1}, y_{1}, z_{1}\right)$ will be acquired by fitting multiple spherical centers. It is obvious to know that the two centers coordinate $C_{0}\left(x_{0}, y_{0}, z_{0}\right)$ and $C_{1}\left(x_{1}, y_{1}, z_{1}\right)$ are not their actual coordinate at world coordinate system. But as the probe is placed near the bottom of elevating axis, the mistakes are relatively small when the two fitting centers coordinate compared to their real value at world coordinate system. In the procedure of calculating vector $\vec{n}\left(d_{x}, d_{y}, d_{z}\right)$, tentatively treat them as true value. Rise the ball to two different heights again, bout $40 \mathrm{~mm}$ and $80 \mathrm{~mm}$ respectively this time, then acquire two circle centers $C_{2}\left(x_{2}, y_{2}, z_{2}\right)$ and $C_{3}\left(x_{3}, y_{3}, z_{3}\right)$ as before, this time the mistakes are relatively large when compared to their true value, so it must be revised to their actual coordinate at world coordinate system, write the actual coordinate as $C_{2}^{\prime}\left(x_{2}^{\prime}, y_{2}^{\prime}, z_{2}^{\prime}\right)$ and $C_{3}^{\prime}\left(x_{3}^{\prime}, y_{3}^{\prime}, z_{3}^{\prime}\right)$. By equation (7) shows that

$$
\left\{\begin{array}{l}
x_{2}^{\prime}=x_{2}+z_{2} d_{x} \\
y_{2}^{\prime}=y_{2}+z_{2} d_{y} \\
z_{2}^{\prime}=z_{2} d_{z} \\
x_{3}^{\prime}=x_{3}+z_{3} d_{x} \\
y_{3}^{\prime}=y_{3}+z_{3} d_{y} \\
z_{3}^{\prime}=z_{3} d_{z}
\end{array}\right.
$$

Since $C_{0}, C_{1}, C_{2}$ and $C_{3}$ are all fitting center points, therefore they are all on $O_{t} Z_{t}$-axis. From straight linespace formula (1):

$$
\left\{\begin{array}{l}
\frac{x_{2}^{\prime}-x_{0}}{x_{1}-x_{0}}=\frac{y_{2}^{\prime}-y_{0}}{y_{1}-y_{0}}=\frac{z_{2}^{\prime}-z_{0}}{z_{1}-z_{0}} \\
\frac{x_{3}^{\prime}-x_{0}}{x_{1}-x_{0}}=\frac{y_{3}^{\prime}-y_{0}}{y_{1}-y_{0}}=\frac{z_{3}^{\prime}-z_{0}}{z_{1}-z_{0}}
\end{array}\right.
$$

Although $\vec{n}\left(d_{x}, d_{y}, d_{z}\right)$ can be computed from equation (9), yet the vector is still imprecise, write it as $\vec{n}_{1}\left(d_{x 1}, d_{y 1}, d_{z 1}\right)$ firstly.

Step 2: Put the ball on the turntable two different heights about $10 \mathrm{~mm}$ and $20 \mathrm{~mm}$ respectively again for scanning, then acquire two circle centers coordinate $C_{4}\left(x_{4}, y_{4}, z_{4}\right)$ and $C_{5}\left(x_{5}, y_{5}, z_{5}\right)$ by fitting spherical centers, utilize equation (7) and vector $\vec{n}_{1}\left(d_{x 1}, d_{y 1}, d_{z 1}\right)$ to compute $C_{4}^{\prime}\left(x_{4}^{\prime}, y_{4}^{\prime}, z_{4}^{\prime}\right)$ and $C_{5}^{\prime}\left(x_{5}^{\prime}, y_{5}^{\prime}, z_{5}^{\prime}\right)$. Therefore, the straight linear equation of $O_{t} Z_{t}$-axis can be determined.

Step 3: If assume the unit vector of elevating axis is $\vec{n}_{2}\left(d_{x 2}, d_{y 2}, d_{z 2}\right)$, put the ball on two different heights to acquire two circle centers coordinate $C_{6}\left(x_{6}, y_{6}, z_{6}\right)$ and $C_{7}\left(x_{7}, y_{7}, z_{7}\right)$ by fitting multiple spherical centers. Utilize equation (7) and vector $\vec{n}_{2}\left(d_{x 2}, d_{y 2}, d_{z 2}\right)$ to compute their actual coordinate $C_{6}^{\prime}\left(x_{6}^{\prime}, y_{6}^{\prime}, z_{6}^{\prime}\right)$ and $C_{7}^{\prime}\left(x_{7}^{\prime}, y_{7}^{\prime}, z_{7}^{\prime}\right)$. Due to $C_{6}^{\prime}$ and $C_{7}^{\prime}$ are all fitting centers, they should be on $O_{t} Z_{t}$-axis, utilize straight line-space formula (1) to obtain:

$$
\left\{\begin{array}{l}
\frac{x_{6}^{\prime}-x_{4}^{\prime}}{x_{5}^{\prime}-x_{4}^{\prime}}=\frac{y_{6}^{\prime}-y_{4}^{\prime}}{y_{5}^{\prime}-y_{4}^{\prime}}=\frac{z_{6}^{\prime}-z_{4}^{\prime}}{z_{5}^{\prime}-z_{4}^{\prime}} \\
\frac{x_{7}^{\prime}-x_{4}^{\prime}}{x_{5}^{\prime}-x_{4}^{\prime}}=\frac{y_{7}^{\prime}-y_{4}^{\prime}}{y_{5}^{\prime}-y_{4}^{\prime}}=\frac{z_{7}^{\prime}-z_{4}^{\prime}}{z_{5}^{\prime}-z_{4}^{\prime}}
\end{array}\right.
$$

From equation (10) can calculate vector $\vec{n}_{2}\left(d_{x 2}, d_{y 2}, d_{z 2}\right)$. Obviously, vector $\vec{n}_{2}$ is more precise than $\vec{n}_{1}$. Through repeated iterative measurements and calculations can eventually acquire a relatively accurate unit vector $\vec{n}\left(d_{x}, d_{y}, d_{z}\right)$ of elevating axis, then utilize equation (7) and $\vec{n}$ to modify all measurement data. 


\section{EXPERIMENTAL RESULTS AND ANALYSIS}

According to the calibration method proposed in this article, the software for calibration and measurement has been developed. Figure. 4 is $3 \mathrm{D}$ point cloud model of a ceramic vane core obtained by the measurement system. For the mechanical errors existing in the system, an iterative data correction method is applied in the measurement. Figure.5 shows the registration result of a ceramic vane core $3 \mathrm{D}$ point cloud compared to standard model as the measurement data without modification, the average error of the measurement results is $0.054 \mathrm{~mm}$. Figure.6 shows the registration result of the 3D point cloud compared to standard model after modifying the measurement data, the average error of the measurement results is $0.031 \mathrm{~mm}$. Judging from the registration accuracy to find that when measurement data after correction, the accuracy of the measurement system received a large increase.

\section{CONCLUSIONS}

In this paper, for a $2 \mathrm{DOF}$ linear structured light $3 \mathrm{D}$ measurement system, a special calibration method is introduced on the basis of high-low ball through fitting multiple spherical centers to obtain a circle center, utilize space vector to determine the position of probe coordinate system relative to turntable coordinate system, and then complete the motion mechanism calibration. In addition, to compensate mechanical errors, an iterative data correction is proposed based on high-low ball measuring principle. After modifying the data, mistakes can be effectively eliminated when measuring. Through the registration result of a ceramic vane core $3 \mathrm{D}$ point cloud compared to standard model, this algorithm can greatly improve the measurement system precision, promote the 2DOF linear structured light 3D measurement system further application in engineering practice.

\section{ACKNOWLEDGMENT}

This work is supported by the China Fundamental Research Funds for the Central Universities, No: 2013 QN047.

\section{REFERENCES}

[1] Zhou Fuqiang, Zhang Guangjun, Jiang Jie. Field calibration method for line structured light vision sensor[J]. Chin. J. Mechanical, Engineering, 2004, 40(6): 169-173.

[2] Song dahu, Li Zhongke, Cheng Chunxia. Research on 3D Coordinate Measurement Technology Based on Line Structured Light[J]. Computer Engineering, 2012, 38(22): 291-292.

[3] Xie Zexiao, Zhang Chengguo, Zhang Guoxiong. Accurate calibration of structured-light sensors for solving the extrinsic parameters $[\mathrm{J}]$. Chinese Journal Mechanical Engineering, 2005,41(8): 218-223.

[4] Weiguang Z, Hong Z, Qi Z. Calibration method for threedimensional measurement system based on linear-structure light $[\mathrm{J}]$. Chinese J. Lasers, 2009, 36(1): 182-188.

[5] Li Yong, Su Xianyu. New method for system calibration in phase measurement profilometry with large view field $[\mathrm{J}]$. Acta Optica Sinica, 2006, 26(8): 1162-1166.

[6] Yu Zujun, Yang Yanan, Zhu Liqiang. Study on Calibration Method for 3D Laser Scanning Systems[J]. Journal of Electronic Measurement and Instrument, 2007, 21(6): 31-35.

[7] Tsai R. A versatile camera calibration technique for high-accuracy 3D machine vision metrology using off-the-shelf TV cameras and lenses[J]. Robotics and Automation, IEEE Journal of, 1987, 3(4): 323-344.

[8] Martins H A, Birk J R, Kelley R B. Camera Models Based on Data from Two Calibration Planes[J]. Computer Graphics and Imaging Processing, 1981, 17(2): 173-180.

[9] Zhang Z. Flexible camera calibration by viewing a plane from unknown orientations[C]. Computer Vision, 1999. The Proceedings of the Seventh IEEE International Conference on. IEEE, 1999, 1: 666-673.

[10] Fu L, Liu Z, Du B. Camera calibration for linear structured light 3D digital measurement system[C]. Intelligent Control an Information Processing (ICICIP), 2010 International Conference on. IEEE, 2010: 352-355.

[11] Wu D, Ma Z, Li A, et al. A novel approach to calibrate a structured light vision sensor in a robot based 3D measurement system $[\mathrm{C}]$ Modelling, Identification and Control (ICMIC), The 2010 International Conference on. IEEE, 2010: 298-303

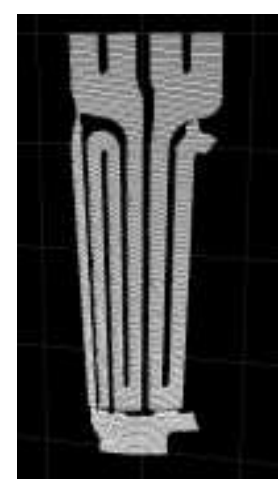

Figure 4. 3D point cloud model of a ceramic vane core

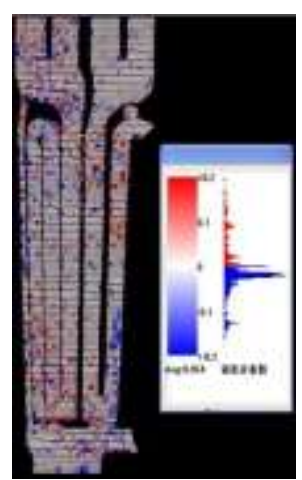

Figure 5. The registration result of the measurement data without modification

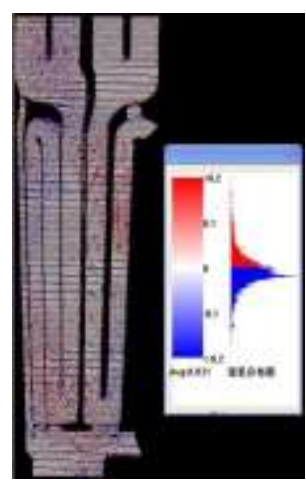

Figure 6. The registration result of the measurement data after modified 